\title{
Factors influencing the food consumption of children and adolescents: a qualitative investigation
}

\author{
A. Fitzgerald ${ }^{1}$, C. Heary ${ }^{1}$, C. Kelly ${ }^{2}$ and E. Nixon ${ }^{3}$ \\ ${ }^{1}$ School of Psychology and ${ }^{2}$ Department of Health Promotion, National University of Ireland, Galway, Republic of Ireland \\ and ${ }^{3}$ School of Psychology, Trinity College Dublin, Republic of Ireland
}

Factors influencing the food choices of children and adolescents need to be better understood in order to develop effective nutrition interventions to improve food consumption patterns. To date, a comprehensive approach to studying factors influencing children's and adolescents' food choices is lacking. In an effort to better understand young people's perceptions of factors influencing their food consumption, qualitative research was conducted with children and adolescents. Schools were randomly selected from the Department of Education and Science published list of schools in the Western region of Ireland. The schools were based in broad catchment areas that reflect a good demographic mix. One youth centre was also selected. Participants were recruited from, and divided into, three distinct age groups (9-10 years, 13-14 years, 16-18 years) covering late-childhood to-late-adolescence, to allow a consideration of the developmental differences in food choice across childhood and adolescence. These age groups were chosen in recognition that as children grow older and become more independent, lifestyle, social, environmental and developmental influences come into play which can change reverse or alter food consumption patterns established in the home ${ }^{(1)}$.

Ethical approval for the study was received from the Research Ethics Committee of the National University of Ireland, Galway. Six semi-structured focus-group discussions of single-gender groups of boys or girls ranging from 9 to 18 years were employed $(N=29)$. Each focus group lasted between 40 to 60 minutes. The inductive thematic analysis method was used for analysis of qualitative data ${ }^{(2)}$. This method permits data analysis to provide a rich and detailed account of data and the themes emerging are strongly linked to the data themselves.

Eight key factors were identified as important influences on food consumption. These factors included food preferences, perceptions of healthy eating, family environment, peer influence, school influence, food advertising, lifestyle characteristics and control \& choice. Within the broader theme of home food environment, sub-themes of family meal patterns, family food practices, home food availability and food purchasing decisions were identified. There was great variation among the focus groups relating to the frequency and occurrence of family meals. Some children reported that family meals were part of a daily routine, whereas for many adolescents, family meals did not occur on a regular basis. Major barriers to having family meals together included busy schedules of parents and adolescents, extracurricular activities, spending time with friends and the adolescents' increased desire for autonomy. Among the adolescents, parental control began to diminish and adolescents gained more independence in relation to their food choices. There were also important developmental differences in influences on food choice between children and adolescents of varying ages. Developmental differences refer to the impact of maturational processes and experience on behaviour. For example, peers appeared to be particularly influential in adolescents' food consumption, however, children did not perceive their friends as an important influence on food choice.

The findings suggest that if programs to improve young people's nutrition are to be effective, they need to gather children's and adolescents' views about the factors influencing their food choices and address a broad range of factors. The findings also support the growing body of literature that suggests that family and social environmental factors play an important role in the development of young people's food consumption patterns ${ }^{(3)}$.

1. Story M \& Neumark-Sztainer D (1999) Promoting healthy eating and physical activity in adolescents. Archive of Pediatric \& Adolescent Medicine, 10, $109-123$.

2. Hayes N (2000) Doing psychological research. Buckingham, England: Open University Press.

3. Patrick H \& Nicklas TA (2005) A review of family and social determinants of children's eating patterns and diet quality. Journal of the American College of Nutrition, 24, 83-92. 